\title{
Long QT syndrome: from channels to cardiac arrhythmias
}

\author{
Arthur J. Moss ${ }^{1}$ and Robert S. Kass ${ }^{2}$
}

\begin{abstract}
${ }^{1}$ Heart Research Follow-up Program, Department of Medicine, University of Rochester School of Medicine and Dentistry, Rochester, New York, USA. 2Department of Pharmacology, Columbia University Medical Center, New York, New York, USA.
\end{abstract}

\begin{abstract}
Long QT syndrome, a rare genetic disorder associated with life-threatening arrhythmias, has provided a wealth of information about fundamental mechanisms underlying human cardiac electrophysiology that has come about because of truly collaborative interactions between clinical and basic scientists. Our understanding of the mechanisms that control the critical plateau and repolarization phases of the human ventricular action potential has been raised to new levels through these studies, which have clarified the manner in which both potassium and sodium channels regulate this critical period of electrical activity.
\end{abstract}

\section{Background}

The common form of long QT syndrome (LQTS), Romano-Ward syndrome (RWS), is a heterogeneous, autosomal dominant, genetic disease caused by mutations of ion channel genes involving the cell membranes of the cardiac myocytes. These channelopathies are associated with delayed ventricular repolarization and are clinically manifest by passing-out spells (syncope; see Glossary) and sudden death from ventricular arrhythmias, notably torsade de pointes (1). Clinically, LQTS is identified by abnormal QT interval prolongation on the ECG. The QT interval prolongation may arise from either a decrease in repolarizing cardiac membrane currents or an increase in depolarizing cardiac currents late in the cardiac cycle. Most commonly, QT interval prolongation is produced by delayed repolarization due to reductions in either the rapidly or the slowly activating delayed repolarizing cardiac potassium $\left(\mathrm{K}^{+}\right)$ current, $\mathrm{I}_{\mathrm{Kr}}$ or $\mathrm{I}_{\mathrm{Ks}}(2)$. Less commonly, QT interval prolongation results from prolonged depolarization due to a small persistent inward "leak" in cardiac sodium $\left(\mathrm{Na}^{+}\right)$current, $\mathrm{I}_{\mathrm{Na}}$ (3) (Figure 1A).

Patients with LQTS are usually identified by QT interval prolongation on the ECG during clinical evaluation of unexplained syncope, as part of a family study when 1 family member has been identified with the syndrome, or in the investigation of patients with congenital neural deafness. The first family with LQTS was reported in 1957 and was thought to be an autosomal recessive disorder (4), but in 1997 it was shown to result from a dominant, homozygous mutation involving the KvLQT1 gene (5), now called the KCNQ1 gene. The more common autosomal dominant RWS was described in 1963-1964, and over 300 different mutations involving 7 different genes (LQT1-LQT7) have now been reported (6). Most of the clinical information currently available regarding LQTS relates to RWS. There is considerable variability in the clinical presentation of LQTS, due to the different genotypes, different mutations, variable penetrance of the mutations, and possibly

Nonstandard abbreviations used: $\beta$-AR, $\beta$-adrenergic receptor; ATS, AndersenTawil syndrome; $\mathrm{I}_{\mathrm{Kr}}$, rapidly activating delayed repolarizing cardiac potassium current; $\mathrm{I}_{\mathrm{Ks}}$, slowly activating delayed repolarizing cardiac potassium current; $\mathrm{I}_{\mathrm{Na}}$, cardiac sodium current; LQTS, long QT syndrome; QTc, heart rate-corrected QT (interval); RWS, Romano-Ward syndrome; TS, Timothy syndrome.

Conflict of interest: The authors have declared that no conflict of interest exists.

Citation for this article: J. Clin. Invest. 115:2018-2024 (2005).

doi:10.1172/JCI25537. genetic and environmental modifying factors. Clinical criteria have been developed to determine the probability of having LQTS, and genotype screening of individuals suspected of having LQTS and of members of known LQTS families has progressively increased the number of subjects with genetically confirmed LQTS. The genes associated with LQTS have been numerically ordered by the chronology of their discovery (LQT1 through LQT7), with $95 \%$ of the known mutations located in the first 3 of the 7 identified LQTS genes (Table 1). All of the LQT genes except LQT4 (ANKB) code for ion channels. LQT4 codes for a protein called ankyrin-B that anchors ion channels to specific domains in the plasma membrane. LQT7 is an ion channel gene, and mutations involving this gene result in a multisystem disease (Andersen-Tawil syndrome) that includes modest QT interval prolongation secondary to reduction in 1 of the potassium repolarization currents (Kir2.1). Prophylactic and preventive therapy for LQTS is directed toward reduction in the incidence of syncope and sudden death and has involved left cervicothoracic sympathetic ganglionectomy, $\beta$-blockers, pacemakers, implanted defibrillators, and gene/mutation-specific pharmacologic therapy (7).

\section{Gene-specific triggers of cardiac arrhythmias}

The discovery that distinct LQTS variants were associated with genes coding for different ion channel subunits has had a major impact on the diagnosis and analysis of LQTS patients. It is clear that there are distinct risk factors associated with the different LQTS genotypes, and this must be taken into account during patient evaluation and diagnosis. The greatest difference in risk factors becomes apparent when LQT3 syndrome patients (SCN5A mutations) are compared with patients with LQT1 syndrome (KCNQ1 mutations) or LQT2 syndrome (KCNH2 mutations). The potential for understanding a mechanistic basis for arrhythmia risk was realized soon after the first genetic information relating mutations in genes coding for distinct ion channels to LQTS became available $(8,9)$ and is still the focus of extensive investigation. This genotype-phenotype association was confirmed in an extensive study in which the risk of cardiac events was studied in LQTS-genotyped patients. This investigation, which focused on patients with KCNQ1 (LQT1), KCNH2 (LQT2), and SCN5A (LQT3) mutations, reported clear differences in arrhythmic risk that was correlated with gene-specific mutations. In the 
A
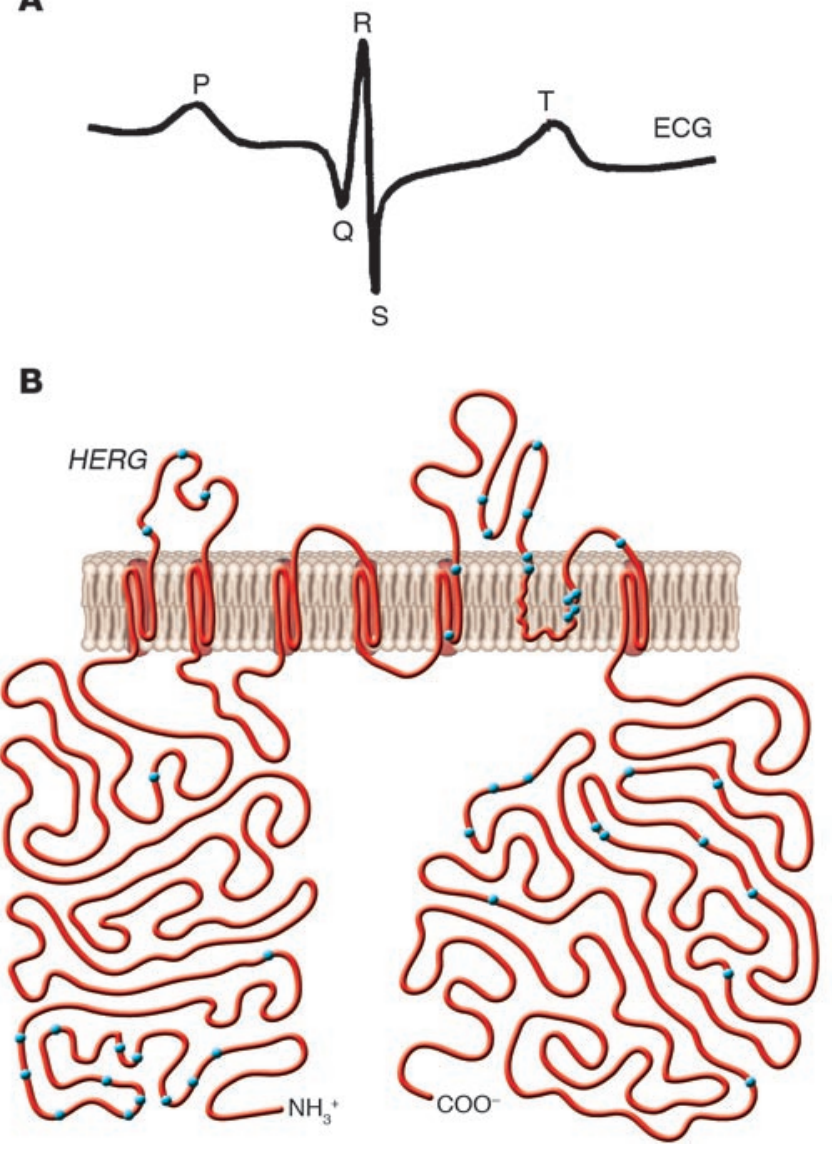

case of SCN5A mutation carriers (LQT3), risk of cardiac events was greatest during rest (bradycardia), when sympathetic nerve activity is expected to be low. In contrast, cardiac events in LQT2 syndrome patients were associated with arousal and/or conditions in which patients were startled, whereas LQT1 syndrome patients were found to be at greatest risk of experiencing cardiac events during exercise or conditions associated with elevated sympathetic nerve activity (10).

Subsequently, additional evidence has linked dysfunctional regulation of the QT interval during exercise to LQT1 mutations (11-13). The contrast between LQT1 and LQT3 patients in the role of adrenergic input and/or heart rate in arrhythmia risk is clear and has raised the possibility of distinct therapeutic strategies in the management of patients with these LQTS variants. In fact, $\beta$-blocker therapy has been shown to be most effective in preventing recurrence of cardiac events and lowering the death rate in LQT1 and LQT2 syndrome patients but is much less effective in the treatment of LQT3 syndrome patients $(14,15)$. $\beta$-Blockers have minimal effects on the heart rate-corrected QT (QTc) interval but are associated with a significant reduction in cardiac events in LQTS patients, probably because these drugs modulate the stimulation of $\beta$-adrenergic receptors ( $\beta$-ARs) and hence the regulation of downstream signaling targets during periods of elevated sympathetic nerve activity. Clinical data for genotyped patients provide strong support for the hypothesis that the effectiveness of $\beta$-blocking drugs depends critically on the genetic basis of the disease; for example, recent data provide evidence that there is still a high rate

\section{Figure 1}

(A) An illustrative example of a single cardiac cycle detected as spatial and temporal electrical gradients on the ECG. The $P$ wave is generated by the spread of excitation through the atria. The QRS complex represents ventricular activation and is followed by the $T$ wave, which reflects ventricular repolarization gradients. (B) Schematic representation of the $K C N H 2$ (HERG) potassium channel $\alpha$ subunit, involving the $\mathrm{N}$-terminal part $\left(\mathrm{NH}_{3}^{+}\right), 6$ membrane-spanning segments with the pore region located from segment S5 to segment S6, and the C-terminal portion ( $\left.\mathrm{COO}^{-}\right)$. Mutation locations are indicated by blue dots. Fourteen different mutations were located in 13 locations within the pore region. Reproduced with permission from Circulation (28).

of cardiac events in LQT2 and LQT3 patients treated with $\beta$-blocking drugs (15). Consequently, even $\beta$-blockers do not provide absolute protection against fatal cardiac arrhythmias.

\section{The sodium channel and mutation-specific pharmacologic therapy}

The SCN5A gene encodes the $\alpha$ subunit of the major cardiac sodium channel (16), and various LQT3 mutations can result in different functional alterations in this channel with similar degrees of QT interval prolongation and cardiac arrhythmias $(17,18)$. For example, the 9-bp deletion with loss of 3 amino acids $(\triangle \mathrm{KPQ})$ in the linker between the third and fourth domains of the $\alpha$ unit of the sodium channel and 3 missense mutations in this gene (N1325S, R1623Q, and R1644H) all promote sustained and inappropriate sodium entry into the myocardial cell during the plateau phase of the action potential, resulting in prolonged ventricular repolarization and the LQTS phenotype. The functional consequences of the D1790G missense mutation are quite different in that this mutation does not promote sustained inward sodium current but, rather, causes a negative shift in steady-state inactivation with a similar LQTS phenotype (19). It should be noted that other mutations in the SCN5A gene can result in Brugada syndrome and conduction system disorders without QT interval prolongation. At least 1 mutation (1795insD) has been shown to have a dual effect, with inappropriate sodium entry at slow heart rates (LQTS ECG pattern) and reduced sodium entry at fast heart rates (Brugada syndrome ECG pattern) (20).

Mutation-specific pharmacologic therapy has been reported in 2 specific SCN5A mutations associated with LQTS. In 1995, Schwartz et al. reported that a single oral dose of the sodium channel blocker mexiletine administered to 7 LQT3 patients with the $\triangle \mathrm{KPQ}$ deletion produced significant shortening of the QTc interval within 4 hours (21). Similar shortening of the QTc interval in LQT3 patients with the $\triangle \mathrm{KPQ}$ deletion has been reported with lidocaine and tocainide (22). Preliminary clinical experience with flecainide revealed normalization of the QTc interval with low doses of this drug in patients with the $\triangle \mathrm{KPQ}$ deletion (23). In 2000, Benhorin et al. reported the effectiveness of open-label oral flecainide in shortening the QTc interval in 8 asymptomatic subjects with the D1790G mutation (24).

The SCN5A-D1790G mutation changes the sodium channel interaction with flecainide. This mutation confers a high sensitivity to use-dependent blockade by flecainide, due in large part to the marked slowing of the repriming of the mutant channels in the presence of the drug (19). Flecainide's tonic block is not affected by the D1790G mutation. These flecainide effects are different from those occurring with the $\triangle \mathrm{KPQ}$ mutant channels (25). 


\section{Table 1}

Nomenclature, gene names, and proteins associated with LQTS

\begin{tabular}{lcc} 
Disease & Gene (historical name) & Protein \\
LQT1 & KCNQ1 (KVLT1) & $\mathrm{I}_{\mathrm{ks}} \mathrm{K}^{+}$channel $\alpha$ subunit \\
LQT2 & KCNH2 (HERG) & $\mathrm{I}_{\mathrm{kr}} \mathrm{K}^{+}$channel $\alpha$ subunit \\
LQT3 & SCN5A & $\mathrm{I}_{\mathrm{Na}} \mathrm{Na}^{+}$channel $\alpha$ subunit \\
LQT4 & ANKB & Ankyrin-B \\
LQT5 & KCNE1 (minK) & $\mathrm{I}_{\mathrm{ks}} \mathrm{K}^{+}$channel $\beta$ subunit \\
LQT6 & KCNE2 (MiRP1) & $\mathrm{I}_{\mathrm{kr}} \mathrm{K}^{+}$channel $\beta$ subunit \\
LQT7 & KCNJ2 & $\mathrm{I}_{\mathrm{Kr} 2.1} \mathrm{~K}^{+}$channel $\alpha$ subunit \\
LQT8 & CACNA1 & Cav1.2 Calcium channel $\alpha$ subunit \\
\hline
\end{tabular}

These flecainide findings in patients with the $\triangle \mathrm{KPQ}$ and D1790G mutations provide encouraging evidence in support of mutationspecific pharmacologic therapy for 2 specific forms of the LQT3 disorder. Larger clinical trials with flecainide in patients with these 2 mutations are needed before this therapy can be recommended as safe and effective for patients with these genetic disorders.

\section{Clinical relevance of mutations in regions of $\mathrm{KCNH} 2$}

The KCNH2 gene encodes the ion channel involved in the rapid component of the delayed rectifier repolarization current $\left(\mathrm{I}_{\mathrm{Kr}}\right)$, and mutations in this gene are responsible for the LQT2 form of LQTS (26). Mutations in KCNH2 are associated with diminution in the repolarizing $\mathrm{I}_{\mathrm{Kr}}$ current with resultant prolongation of ventricular repolarization and lengthening of the QT interval. During the 1990s, it was appreciated that several drugs, such as terfenadine and cisapride, caused QT interval prolongation by reducing $\mathrm{I}_{\mathrm{Kr}}$ current through the pore region of the KCNH2 channel (27). These findings raised the question of whether mutations in the pore region of the KCNH2 channel would be associated with a more virulent form of LQT2 than mutations in non-pore regions.

In a report from the International LQTS Registry, 44 different KCNH2 mutations were identified in 201 subjects, with 14 mutations in 13 locations in the pore region (amino acid residues 550-650) (Figure 1B) (28). Thirty-five subjects had mutations in the pore region and 166 in non-pore regions. When birth was used as the time origin with follow-up through age 40, subjects with pore mutations had more severe clinical manifestations of the genetic disorder and experienced a higher frequency of arrhythmia-related cardiac events at an earlier age than did subjects with non-pore mutations. The cumulative probability of a first cardiac event before $\beta$-blockers were initiated in subjects with pore mutations and non-pore mutations in the $\mathrm{KCNH} 2$ channel is shown in Figure 2, with a hazard ratio in the range of $11(P<0.0001)$ at an adjusted QTC interval of 0.50 seconds. This study involved a limited number of different $\mathrm{KCNH} 2$ mutations and only a small number of subjects with each mutation. Missense mutations made up $94 \%$ of the pore mutations, and thus it was not possible to evaluate risk by the mutation type within the pore region.

These findings indicate that mutations in different regions of the KCNH2 potassium channel can be associated with different levels of risk for cardiac arrhythmias in LQT2. An important question is whether similar region-related risk phenomena exist in the other LQTS channels. Two studies evaluated the clinical risk of mutations located in different regions of the KCNQ1 (LQT1) gene and reported contradictory findings. One study found no significant differences in clinical presentation, ECG parameters, and cardiac events among 294 LQT1 patients with KCNQ1 mutations located in the pre-pore region including the $\mathrm{N}$-terminus (1 to 278 ), the pore region (279 to 354$)$, and the post-pore region including the C-terminus (>354) (29). In contrast, another study of 66 LQT1 patients found that mutations in the transmembrane portion of KCNQ1 were associated with a higher risk of LQTSrelated cardiac events and had greater sensitivity to sympathetic stimulation than mutations located in the $\mathrm{C}$-terminal region (30). These different findings in the 2 LQT1 studies may reflect, in part, population-related genetic heterogeneity, since 1 population was almost entirely white and the subjects in the other study were Japanese. Much larger homogeneous populations need to be studied to resolve this issue.

\section{Insights gained from interactions of clinical and basic scientists}

Though a rare congenital disorder, LQTS has provided a wealth of information about fundamental mechanisms underlying human cardiac electrophysiology that has come about because of truly collaborative interactions between clinical and basic scientists. Our understanding of the mechanisms that control the critical plateau and repolarization phases of the human ventricular action potential (Figure 3) has been raised to new levels through these studies with clarification about the manner in which both potassium and sodium channels regulate this critical period of electrical activity.

\section{Potassium channel currents and the action potential plateau: the delayed rectifiers}

It had been known since 1969 that potassium currents with unique kinetic and voltage-dependent properties were important to the cardiac action potential plateau $(31,32)$. Because of the unique voltage dependence, these currents were referred to as delayed rectifiers. In a pivotal study, Sanguinetti and Jurkiewicz used a pharmacologic analysis to demonstrate 2 distinct components of the delayed rectifier potassium current in the heart: $\mathrm{I}_{\mathrm{Kr}}$ and $\mathrm{I}_{\mathrm{Ks}}(33)$. The $\mathrm{I}_{\mathrm{Ks}}$ component had previously been shown to be under control

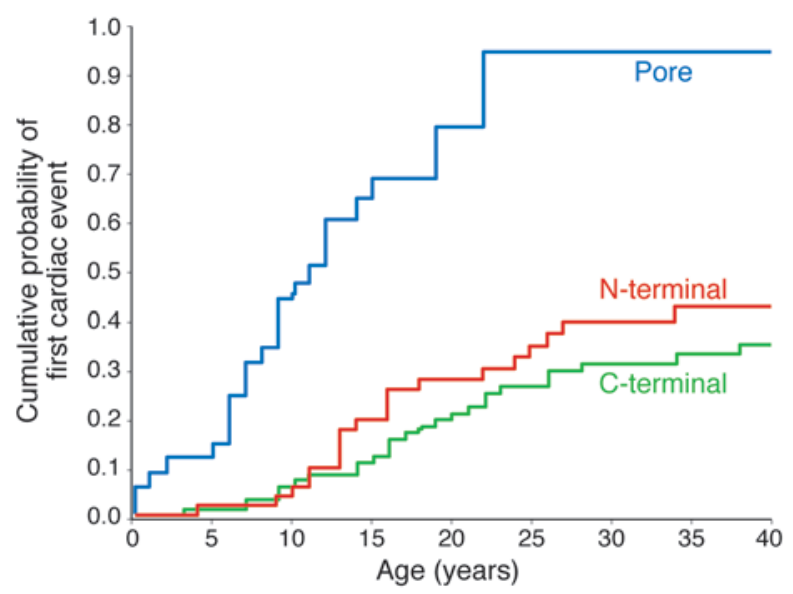

Figure 2

Kaplan-Meier cumulative probability of first cardiac events from birth through age 40 years for subjects with mutations in pore $(n=34)$, $\mathrm{N}$-terminal $(n=54)$, and C-terminal $(n=91)$ regions of the $\mathrm{KCNH} 2$ (HERG) channel. The curves are significantly different $(P<0.0001$, log-rank), mainly because of the high first-event rate in subjects with pore mutations. Reproduced with permission from Circulation (28). 


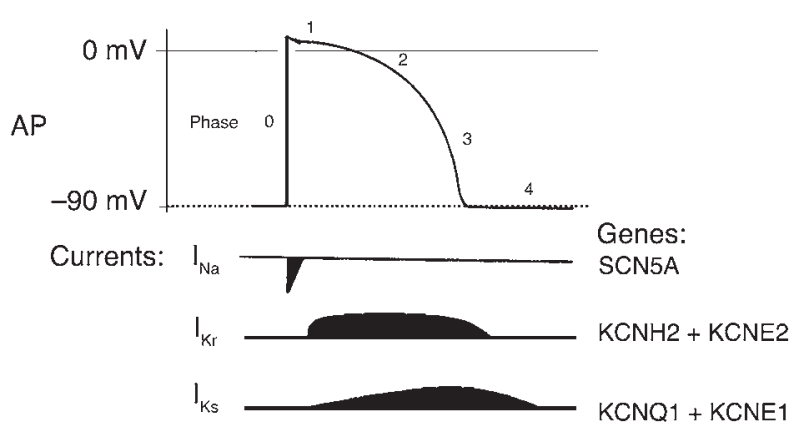

Figure 3

Three major cardiac ion channel currents $\left(\mathrm{I}_{\mathrm{Na}}, \mathrm{I}_{\mathrm{Kr}}\right.$, and $\left.\mathrm{I}_{\mathrm{Ks}}\right)$ and respective genes responsible for generation of portions of the ventricular action potential (AP).

of the sympathetic nervous system, providing an increase in repolarization currents in the face of $\beta$-AR agonists in cellular models (34), but the molecular identity and the relevance to human electrophysiology were not only unclear, but controversial. The clear clinical importance and the genetic basis of these potassium currents were revealed through LQTS investigations.

The first report linking potassium channel dysfunction to LQTS revealed the molecular identity of 1 of the delayed rectifier channels and confirmed the pharmacologic evidence for independent channels underlying these currents (35). This ground-breaking study, a collaborative effort between clinical and basic scientists, revealed that $\mathrm{KCNH} 2$ encodes the $\alpha$ (pore-forming) subunit of the $\mathrm{I}_{\mathrm{Kr}}$ channel and that the rectifying properties of this channel, identified previously by pharmacologic dissection, are indigenous to the channel protein. This work not only provided the first clear evidence for a role of this channel in the congenital LQTS but also laid the base line for future studies that would show that it is the KCNH2 channel that underlies almost all cases of acquired LQTS (36).

The next breakthrough came in 1996 when a collaborative clinical/basic effort discovered that LQTS variant 1 (LQT1) was caused by mutations in a gene (KCNQ1) coding for an unusual potassium channel subunit that could be studied in heterologous expression systems (37). Basic studies almost immediately revealed the functional role of the KCNQ1 gene product: the $\alpha$ (pore-forming) subunit of the $\mathrm{I}_{\mathrm{Ks}}$ channel $(38,39)$. Furthermore, these studies indicated that a previously reported but as-yet poorly understood gene (KCNE1) formed a key regulatory subunit of this important channel. Mutations in KCNE1 have subsequently been linked to LQT5 (40). Now the molecular identity of the 2 cardiac delayed rectifiers had been established.

As is summarized above, clinical studies had provided convincing evidence linking sympathetic nerve activity and arrhythmia susceptibility in LQTS patients, particularly in patients harboring LQT1 mutations. These data and previous basic reports of the robust sensitivity of the slow delayed rectifier component, $\mathrm{I}_{\mathrm{Ks}}$, to $\beta$-AR agonists (34) motivated investigation of the molecular links between KCNE1 channels and $\beta$-AR stimulation, which revealed, for the first time, that the KCNQ1/KCNE1 channel is part of a macromolecular signaling complex in the human heart (41). The channel complexes with an adaptor protein called Yotiao that in turn directly binds key enzymes in the $\beta$-AR signaling cascade (protein kinase A and protein phosphatase 1) and recruits them to form a local signaling environment to control the phosphorylation state of the channel. Mutations in either KCNQ1 (41) or KCNE1 (42) can disrupt this regulation and create heterogeneity in the cellular response to $\beta$-AR stimulation, a novel mechanism that may contribute to the triggering of some arrhythmias in LQT1 and LQT5 (43). This fundamental work is a direct consequence of collaborative efforts between basic and clinical investigators.

\section{Trafficking and its pharmacologic rescue as a mechanism in LQT2}

LQT2 mutations reduce repolarizing current through HERG channels, contributing to delayed repolarization of the ventricular action potential and the resulting QT interval. Though all LQT2 mutations lead to the same clinical phenotype, prolonged QT intervals, multiple mechanisms at the level of the channel protein, including altered gating kinetics and dominant-negative effects via subunit assembly, may underlie this condition.

January and colleagues first reported that mutation-altered protein trafficking could also cause LQT2 (44). Trafficking defects reduce the number of functional channels expressed in the sarcolemmal membrane, contributing to a reduction in repolarizing current, but cause no alteration in the basic biophysical properties of those channels that are functional. Importantly, and uniquely, January and colleagues also made the important discovery that mutation-induced trafficking defects could be restored by temperature and incubation with pharmacologic agents that block HERG channels (45). These observations have subsequently been confirmed by multiple groups and for several distinct LQT2 mutations (46-48), and, most recently, experimental work has suggested that these LQT2 mutations may cause subtle protein misfolding and retention in the endoplasmic reticulum and that drug binding might act as a chaperone to restore maturation efficiency and hence a higher level of sarcolemmal channel protein (49).

\section{Insights into cardiac sodium channels}

The report that mutations in SCN5A, the gene coding for the $\alpha$ subunit of the major cardiac sodium channel, were associated with LQTS (17) was surprising, because this channel is associated most frequently with impulse conduction and hence the QRS but not the QT waveforms of the ECG. Sodium channels are voltage-gated channels that rapidly enter a nonconducting inactivated state during sustained depolarization such as the cardiac action potential plateau. Importantly, the first SCN5A mutation, the $\triangle$ KPQ mutation, physically disrupted a cytoplasmic peptide linker in the channel protein that, in basic biochemical and biophysical studies, had been shown to be a critical determinant of sodium channel inactivation: the inactivation gate $(50,51)$. This peptide links 2 domains (III and IV) of the channel and physically moves to occlude the channel pore upon depolarization. Once again, the combination of basic and clinical investigation has led to a clear understanding of the molecular basis of this key physiological parameter in the human heart. Further, the demonstration that small changes in sodium channel inactivation such at those changes that occur in LQT3 mutations can have life-threatening consequences confirms predictions made more than 50 years ago when it was shown that the cardiac action potential plateau was an exquisitely sensitive period of electrical activity that could adapt, with little energy expenditure, to small changes in ionic currents (52).

Subsequent investigations of LQT3 mutations have revealed that not only is the domain III/IV intracellular linker key to inac- 


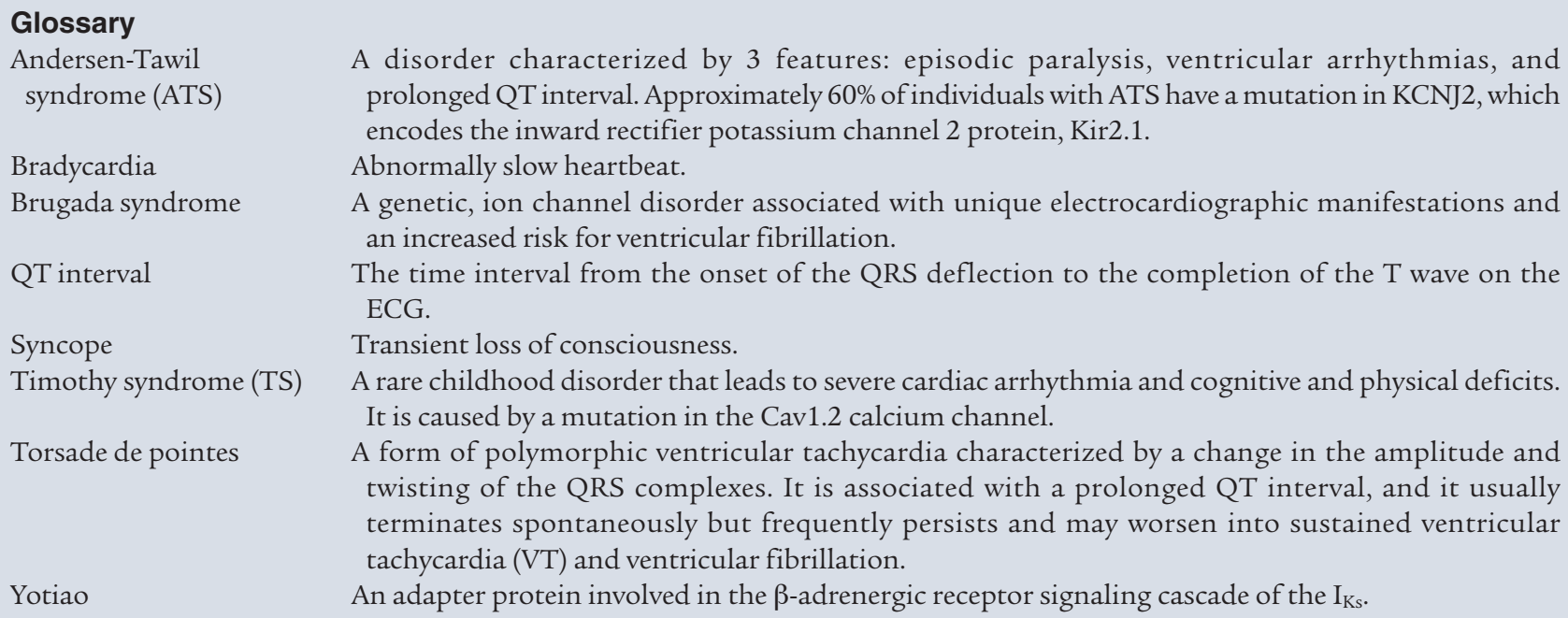

A disorder characterized by 3 features: episodic paralysis, ventricular arrhythmias, and prolonged QT interval. Approximately $60 \%$ of individuals with ATS have a mutation in KCNJ2, which encodes the inward rectifier potassium channel 2 protein, Kir2.1.

Abnormally slow heartbeat.

A genetic, ion channel disorder associated with unique electrocardiographic manifestations and an increased risk for ventricular fibrillation.

The time interval from the onset of the QRS deflection to the completion of the T wave on the ECG.

Transient loss of consciousness.

A rare childhood disorder that leads to severe cardiac arrhythmia and cognitive and physical deficits. It is caused by a mutation in the Cav1.2 calcium channel.

A form of polymorphic ventricular tachycardia characterized by a change in the amplitude and twisting of the QRS complexes. It is associated with a prolonged QT interval, and it usually terminates spontaneously but frequently persists and may worsen into sustained ventricular tachycardia (VT) and ventricular fibrillation.

An adapter protein involved in the $\beta$-adrenergic receptor signaling cascade of the $\mathrm{I}_{\mathrm{Ks}}$.

tivation and maintenance of the action potential plateau (and hence QT interval), but also the channel carboxyterminal (C-T) domain is essential in this process. In fact, evidence generated by basic studies of LQT3 mutations has now shown that the C-T domain forms a molecular complex with the inactivation gate that is necessary to stabilize the nonconducting state of the channel during prolonged depolarization (53), and, further, that this interaction may be modulated by cell calcium and calmodulin (54). Thus there is the intriguing possibility that dysfunction in sodium channel inactivation that can lead to QT interval prolongation may occur in other forms of human cardiovascular pathology when regulation of cellular calcium concentration may be disrupted. Thus, once again, collaboration between clinical and basic scientists investigating a rare inherited disorder has resulted in fundamentally novel insight into cardiovascular physiology, which may have wide-ranging impact on our understanding of human cardiovascular disease.

\section{LQTS variants and multisystem disorders}

Andersen-Tawil syndrome (ATS) is a skeletal muscle and cardiac muscle disease. Clinical phenotypes of patients with ATS include periodic paralysis, developmental dysmorphisms, and prolonged QT intervals. Mutations in KCNJ2 were found to underlie this multisystem disorder (55-57). KCNJ2 encodes the inward rectifier $\mathrm{K}^{+}$ channel $\alpha$ subunit Kir2.1, which is expressed in skeletal and cardiac muscle. Interestingly, like mutations in HERG described above, at least a subset of ATS KCNJ2 mutations have been shown to cause trafficking defects, and hence loss of functional channel activity, of inwardly rectifying potassium channels (58). These genetic and clinical data have confirmed the key role of this inwardly rectifying potassium channel not only in skeletal muscle physiology, but also in control of the duration of the ventricular action potential, a role not often associated with this ionic pathway.

Most recently, a second multiorgan syndrome, Timothy syndrome (TS), has been reported. Like ATS, clinical phenotypes of patients with TS include a wide variety of organ dysfunction, including lethal arrhythmias, webbing of fingers and toes, autism, immune deficiency, intermittent hypoglycemia, cognitive abnormalities, and markedly prolonged QT intervals on the ECG (59).
Importantly, this multisystem dysfunction was found to be caused by mutations in a splice variant of the gene (CACNA1I) coding for the $\alpha$ subunit (Cav1.2) of the L-type calcium channel. The Cav1.2 channel is a principal pathway for calcium entry in cells in which it is expressed, and, in the heart, its activation and inactivation are critical regulators of action potential waveforms and activation of contraction. Hence it is not surprising that mutations of Cav1.2 cause marked clinical dysfunction. Like LQT3 mutations, the TS mutations disrupt inactivation of Cav1.2 channels, but unlike voltage-gated sodium channels, inactivation of Cav1.2 channels is regulated by both voltage and calcium entry (60), and the TS mutations almost completely abolish the voltage-dependent inactivation component. Thus, as has been the case with all other LQTS-related mutations, the combination of clinical and basic investigations of TS patients and mutations will provide the clearest insight to date into the functional roles of these biophysical channel properties in the human heart.

\section{LQT4 is caused by mutations in an adaptor protein}

In 1995, Schott et al. reported a study of a 65-member family in which LQTS was also associated with marked sinus bradycardia (61). Genetic linkage was observed for a region of chromosome 4 located in the interval $4 q 25-q 27$, a region that did not contain candidate ion channel genes, and the identification of the genetic basis of variant LQT4 remained elusive. A clue to the basis of this variant emerged from a study by Chauhan et al. in which sodium channel activity in mice lacking ankyrin-B - not an ion channel protein, but an adaptor protein that associated with cytoskeletal interactions - was altered (62). The major form of ankyrin-B in cardiac cells is $220 \mathrm{kDa}$. Ankyrins typically contain 3 functional domains that consist of the membrane-binding domain, the spectrin-binding domain, and the regulatory domain. Ankyrins bind to multiple proteins that can contribute (directly or indirectly) to cardiac electrical activity. These include the anion exchanger $\left(\mathrm{Cl}^{-} /\right.$ $\mathrm{HCO}_{3}{ }^{-}$exchanger), the $\mathrm{Na}^{+}, \mathrm{K}^{+}$-ATPase, voltage-sensitive sodium channels, the $\mathrm{Na}^{+} / \mathrm{Ca}^{2+}$ exchanger $\left(\mathrm{NCX}\right.$, or $\mathrm{I}_{\mathrm{Na}-\mathrm{Ca}}$ ), and calciumrelease channels including those mediated by ryanodine or inositol trisphosphate $\left(\mathrm{IP}_{3}\right)$ receptors $(63)$. The identify of the LQT4 gene as the gene coding for ankyrin-B was confirmed by Mohler 
et al., who reported a loss of function in the ankyrin-B mutation (E1425G) that causes dominantly inherited type 4 long QT cardiac arrhythmia in humans (64). Physiological insight into the mechanistic basis for LQT4 arrhythmias came from genetically altered mice heterozygous for a null mutation in ankyrin-B. Because of the multiple proteins that associate with ankyrin- $B$, the results indicated that these arrhythmias may develop because of a combined disruption in the cellular organization of the sodium pump, the sodium/calcium exchanger, and inositol-1,4,5-trisphosphate receptors. Interestingly, the ankyrin-B mutation also leads to altered $\mathrm{Ca}^{2+}$ signaling in adult cardiomyocytes, which plays a major role in the triggering of arrhythmias in this mouse model (64). Recently, further ankyrin-B mutations have been reported to be causally linked to LQTS (65). The mechanistic basis of LQT4associated arrhythmias is complex, but, as is the case for TS, it will provide important insight into the pivotal role played by the regulation of intracellular calcium in the genesis and prevention of cardiac arrhythmias in humans.

1. Moss, A.J., et al. 1991. The long QT syndrome: prospecitve longitudinal study of 328 families. Circulation. 84:1136-1144.

2. Sanguinetti, M.C., and Zou, A. 1997. Molecular physiology of cardiac delayed rectifier $\mathrm{K}+$ channels [review]. Heart Vessels. 12(Suppl.):170-172.

3. Bennett, P.B., Yazawa, K., Makita, N., and George, A.L. 1995. Molecular mechanism for an inherited cardiac arrhythmia. Nature. 376:683-685.

4. Jervell, A., and Lange-Nielsen, F. 1957. Congenital deaf-mutism, functional heart disease with prolongation of the Q-T interval and sudden death. Am. Heart J. 54:59-68.

5. Splawski, I., Timothy, K.W., Vincent, G.M., Atkinson, D.L., and Keating, M.T. 1997. Molecular basis of the long-QT syndrome associated with deafness. N. Engl. J. Med. 336:1562-1567.

6. Splawski, I., et al. 2000. Spectrum of mutations in long-QT syndrome genes: KVLQT1, HERG, SCN5A, KCNE1, and KCNE2. Circulation. 102:1178-1185.

7. Moss, A.J. 2003. Long QT syndrome. JAMA. 289:2041-2044.

8. Priori, S.G., Napolitano, C., Paganini, V., Cantu, F., and Schwartz, P.J. 1997. Molecular biology of the long QT syndrome: impact on management. Pacing Clin. Electrophysiol. 20:2052-2057.

9. Zareba, W., et al. 1998. Influence of genotype on the clinical course of the long-QT syndrome. International Long-QT Syndrome Registry Research Group. N. Engl. J. Med. 339:960-965.

10. Schwartz, P.J., et al. 2001. Genotype-phenotype correlation in the long-QT syndrome: gene-specific triggers for life-threatening arrhythmias. Circulation. 103:89-95.

11. Ackerman, M.J., et al. 2002. Epinephrine-induced QT interval prolongation: a gene-specific paradoxical response in congenital long QT syndrome. Mayo Clin. Proc. 77:413-421.

12. Takenaka, K., et al. 2003. Exercise stress test amplifies genotype-phenotype correlation in the LQT1 and LQT2 forms of the long-QT syndrome. Circulation. 107:838-844.

13. Paavonen, K.J., et al. 2001. Response of the QT interval to mental and physical stress in types LQT1 and LQT2 of the long QT syndrome. Heart. 86:39-44.

14. Moss, A.J., et al. 2000. Effectiveness and limitations of beta-blocker therapy in congenital long-QT syndrome. Circulation. 101:616-623.

15. Priori, S.G., et al. 2004. Association of long QT syndrome loci and cardiac events among patients treated with beta-blockers. JAMA. 292:1341-1344.

16. George, A.L., et al. 1995. Assignment of the human

\section{Conclusions}

We have made considerable progress in understanding the importance of ion channel structure to human physiology since the first ion channel was cloned in 1982. We now have a better understanding of the molecular genetics, ion channel structures, and cellular electrophysiology that contribute to the genesis of cardiac arrhythmias. Much of this improved insight has come directly from investigations of LQTS and other inherited arrhythmias and is being translated into more effective and more rational therapy for patients with electrical disorders of the cardiac rhythm. Much remains to be accomplished, and this will be done through continued collaboration of basic and clinical scientists based on the foundations laid by studies of LQTS.

Address correspondence to: Arthur J. Moss, Box 653, University of Rochester Medical Center, Rochester, New York 14642, USA. Phone: (585) 275-5391; Fax: (585) 273-5283; E-mail: heartajm@ heart.rochester.edu. heart tetrodotoxin-resistant voltage-gated $\mathrm{Na}+$ channel alpha-subunit gene (SCN5A) to band 3P21. Cytogenet. Cell Genet. 68:67-70.

17. Wang, Q., et al. 1995. SCN5A mutations associated with an inherited cardiac arrhythmia, long QT syndrome. Cell. 80:805-811.

18. Wang, Q., et al. 1995. Cardiac sodium channel mutations in patients with long QT syndrome, an inherited cardiac arrhythmia. Hum. Mol. Genet. 4:1603-1607.

19. Abriel, H., Wehrens, X.H., Benhorin, J., Kerem, B., and Kass, R.S. 2000. Molecular pharmacology of the sodium channel mutation D1790G linked to the long-QT syndrome. Circulation. 102:921-925.

20. Veldkamp, M.W., et al. 2000. Two distinct congenital arrhythmias evoked by a multidysfunctional $\mathrm{Na}(+)$ channel. Circ. Res. 86:E91-E97.

21. Schwartz, P.J., et al. 1995. Long QT syndrome patients with mutations of the SCN5A and HERG genes have differential responses to NA+ channel blockade and to increases in heart rate: implications for gene-specific therapy. Circulation. 92:3381-3386.

22. Rosero, S.Z., Zareba, W., Robinson, J.L., and Moss, A. 1997. Gene-specific therapy for long QT syndrome: QT shortening with lidocaine and tocainide in patients with mutation of the sodium channel gene. Ann. Noninvasive Electrocardiol. 2:274-278.

23. Windle, J.R., Geletka, R.C., Moss, A.J., Zareba, W., and Atkins, D.L. 2001. Normalization of ventricular repolarization with flecainide in long QT syndrome patients with SCN5A:DeltaKPQ mutation. Ann. Noninvasive Electrocardiol. 6:153-158.

24. Benhorin, J., et al. 2000. Effects of flecainide in patients with new SCN5A mutation: mutationspecific therapy for long-QT syndrome? Circulation. 101:1698-1706.

25. Nagatomo, T., January, C.T., and Makielski, J.C. 2000. Preferential block of late sodium current in the LQT3 DeltaKPQ mutant by the class I(C) antiarrhythmic flecainide. Mol. Pharmacol. 57:101-107.

26. January, C.T., Gong, Q., and Zhou, Z. 2000. Long QT syndrome: cellular basis and arrhythmia mechanism in LQT2. J. Cardiovasc. Electrophysiol. 11:1413-1418.

27. Sanguinetti, M.C., Curran, M.E., Spector, P.S., and Keating, M.T. 1996. Spectrum of HERG K channel dysfunction in an inherited cardiac arrhythmia. Proc. Natl. Acad. Sci. U. S. A. 93:2208-2212.

28. Moss, A.J., et al. 2002. Increased risk of arrhythmic events in long-QT syndrome with mutations in the pore region of the human ether-a-go-go-related gene potassium channel. Circulation. 105:794-799.

29. Zareba, W., et al. 2003. Location of mutation in the KCNQ1 and phenotypic presentation of long QT syndrome. J. Cardiovasc. Electrophysiol. 14:1149-1153.

30. Shimizu, W., et al. 2004. Mutation site-specific differences in arrhythmic risk and sensitivity to sympathetic stimulation in the LQT1 form of congenital long QT syndrome: multicenter study in Japan. J. Am. Coll. Cardiol. 44:117-125.

31. Noble, D., and Tsien, R. 1968. The kinetics and rectifier properties of the slow potassium current in cardiac Purkinje fibres. J. Physiol. 195:185-214.

32. Noble, D., and Tsien, R.W. 1969. Outward membrane currents activated in the plateau range of potentials in cardiac Purkinje fibres. J. Physiol. 200:205-231.

33. Sanguinetti, M.C., and Jurkiewicz, N.K. 1990. Two components of cardiac delayed rectifier $\mathrm{K}+$ current. Differential sensitivity to block by class III antiarrhythmic agents. J. Gen. Physiol. 96:195-215.

34. Kass, R.S., and Wiegers, S.E. 1982. The ionic basis of concentration-related effects of noradrenaline on the action potential of calf cardiac purkinje fibres. J. Physiol. 322:541-558.

35. Sanguinetti, M.C., Jiang, C., Curran, M.E., and Keating, M.T. 1995. A mechanistic link between an inherited and an acquired cardiac arrhythmia: HERG encodes the IKr potassium channel. Cell. 81:299-307.

36. Mitcheson, J.S., Chen, J., Lin, M., Culberson, C., and Sanguinetti, M.C. 2000. A structural basis for druginduced long QT syndrome. Proc. Natl. Acad. Sci. U. S. A. 97:12329-12333.

37. Wang, Q., et al. 1996. Positional cloning of a novel potassium channel gene: KVLQT1 mutations cause cardiac arrhythmias. Nat. Genet. 12:17-23.

38. Sanguinetti, M.C., et al. 1996. Coassembly of KvLQT1 and $\operatorname{minK}($ ISK) proteins to form cardiac IKS potassium channel. Nature. 384:80-83.

39. Barhanin, J., et al. 1996. K(V)LQT1 and lsK (minK) proteins associate to form the $\mathrm{I}(\mathrm{Ks})$ cardiac potassium current. Nature. 384:78-80.

40. Splawski, I., Tristani-Firouzi, M., Lehmann, M.H., Sanguinetti, M.C., and Keating, M.T. 1997. Mutations in the hminK gene cause long QT syndrome and suppress IKs function. Nat. Genet. 17:338-340.

41. Marx, S.O., et al. 2002. Requirement of a macromolecular signaling complex for beta adrenergic receptor modulation of the KCNQ1-KCNE1 potassium channel. Science. 295:496-499.

42. Kurokawa, J., Chen, L., and Kass, R.S. 2003. Requirement of subunit expression for cAMPmediated regulation of a heart potassium channel. Proc. Natl. Acad. Sci. U. S. A. 100:2122-2127.

43. Kass, R.S., and Moss, A.J. 2003. Long QT syn- 
drome: novel insights into the mechanisms of cardiac arrhythmias. J. Clin. Invest. 112:810-815. doi:10.1172/JCI200319844.

44. Furutani, M., et al. 1999. Novel mechanism associated with an inherited cardiac arrhythmia: defective protein trafficking by the mutant HERG (G601S) potassium channel. Circulation. 99:2290-2294.

45. Zhou, Z., Gong, Q., and January, C.T. 1999. Correction of defective protein trafficking of a mutant HERG potassium channel in human long QT syndrome. Pharmacological and temperature effects. J. Biol. Chem. 274:31123-31126.

46. Ficker, E., Obejero-Paz, C.A., Zhao, S., and Brown, A.M. 2002. The binding site for channel blockers that rescue misprocessed human long QT syndrome type 2 ether-a-gogo-related gene (HERG) mutations. J. Biol. Chem. 277:4989-4998.

47. Paulussen, A., et al. 2002. A novel mutation (T65P) in the PAS domain of the human potassium channel HERG results in the long QT syndrome by trafficking deficiency. J. Biol. Chem. 277:48610-48616.

48. Rajamani, S., Anderson, C.L., Anson, B.D., and January, C.T. 2002. Pharmacological rescue of human $\mathrm{K}(+)$ channel long-QT2 mutations: human ethera-go-go-related gene rescue without block. Circulation. 105:2830-2835.

49. Gong, Q., Anderson, C.L., January, C.T., and Zhou, Z. 2004. Pharmacological rescue of trafficking defective HERG channels formed by coassembly of wild-type and long QT mutant N470D subunits. Am. J. Physiol. Heart Circ. Physiol. 287:H652-H658.

50. Stuhmer, W., et al. 1989. Structural parts involved in activation and inactivation of the sodium channel. Nature. 339:597-603.

51. Catterall, W.A. 1995. Structure and function of voltage-gated ion channels [review]. Annu. Rev. Biochem. 64:493-531.

52. Weidmann, S. 1952. The electrical constants of Purkinje fibres. J. Physiol. 118:348-360.

53. Motoike, H.K., et al. 2004. The $\mathrm{Na}+$ channel inactivation gate is a molecular complex: a novel role of the COOH-terminal domain. J. Gen. Physiol. 123:155-165.

54. Kim, J., et al. 2004. Calmodulin mediates Ca2+ sensitivity of sodium channels. J. Biol. Chem. 279:45004-45012.

55. Donaldson, M.R., Yoon, G., Fu, Y.H., and Ptacek, L.J. 2004. Andersen-Tawil syndrome: a model of clinical variability, pleiotropy, and genetic heterogeneity. Ann. Med. 36(Suppl. 1):92-97.

56. Andelfinger, G., et al. 2002. KCNJ2 mutation results in Andersen syndrome with sex-specific cardiac and skeletal muscle phenotypes. Am. J. Hum. Genet. 71:663-668.

57. Tristani-Firouzi, M., et al. 2002. Functional and clinical characterization of KCNJ2 mutations associated with LQT7 (Andersen syndrome). J. Clin. Invest. 110:381-388. doi:10.1172/JCI200215183.

58. Bendahhou, S., et al. 2003. Defective potassium channel Kir2.1 trafficking underlies AndersenTawil syndrome. J. Biol. Chem. 278:51779-51785.

59. Splawski, I., et al. 2004. Ca(V)1.2 calcium channel dysfunction causes a multisystem disorder including arrhythmia and autism. Cell. 119:19-31.

60. Kass, R.S., and Sanguinetti, M.C. 1984. Calcium channel inactivation in the cardiac Purkinje fiber. Evidence for voltage- and calcium-mediated mechanisms. J. Gen. Physiol. 84:705-726.

61. Schott, J.J., et al. 1995. Mapping of a gene for long QT syndrome to chromosome 4q25-27. Am. J. Hum. Genet. 57:1114-1122.

62. Chauhan, V.S., Tuvia, S., Buhusi, M., Bennett, V., and Grant, A.O. 2000. Abnormal cardiac $\mathrm{Na}(+)$ channel properties and QT heart rate adaptation in neonatal ankyrin(B) knockout mice. Circ. Res. 86:441-447.

63. Yong, S., Tian, X., and Wang, Q. 2003. LQT4 gene: the "missing" ankyrin. Mol. Interv. 3:131-136.

64. Mohler, P.J., et al. 2003. Ankyrin-B mutation causes type 4 long-QT cardiac arrhythmia and sudden cardiac death. Nature. 421:634-639.

65. Mohler, P.J., et al. 2004. A cardiac arrhythmia syndrome caused by loss of ankyrin-B function. Proc. Natl. Acad. Sci. U. S. A. 101:9137-9142. 